\title{
Scaling Laws of Sum Rate Using Time-Sharing, DPC, and Beamforming for MIMO Broadcast Channels
}

\author{
Masoud Sharif and Babak Hassibi ${ }^{1}$ \\ Dept. Electrical Engineering \\ California Institute of Technology, Pasadena, CA 91125 \\ \{masoud, hassibi\}@systems. caltech.edu
}

\begin{abstract}
We derive the scaling laws of the sum rate throughput for MIMO Gaussian broadcast channels using time-sharing to the strongest user, dirty paper coding (DPC), and beamforming when the number of users (receivers) $n$ is large. Assuming a fixed total average transmit power, we show that for a system with $M$ transmit antennas and users equipped with $N$ antennas, the sum rate scales like $M \log \log n N$ for DPC and beamforming when $M$ is fixed and for any $N$ (either growing to infinity or not). On the other hand, when both $M$ and $N$ are fixed, the sum rate of time-sharing to the strongest user scales like $\min (M, N) \log \log n$. It is also shown that if $M$ grows as $\log n$, the sum rate of DPC and beamforming will grow linearly in $M$, but with different constant multiplicative factors. In this region, the sum rate capacity of time-sharing scales like $N \log \log n$.
\end{abstract}

\section{INTRODUCTION}

With full channel state information (CSI) at the transmitter and the receivers, the MIMO Gaussian broadcast channel is not degraded. In $[1,2,3,4]$, it is proved that the sum rate capacity for such broadcast channels is achieved by using dirty paper coding (DPC). On the other hand, beamforming has long been used for down-link scheduling in MIMO broadcast systems as a heuristic method to reduce the interference in the system. Furthermore, for the single antenna broadcast channel, if the transmitter has full CSI, we can maximize the sum rate by using time division multiplexing and transmitting to the best user (we call this scheduling time-sharing).

\section{Channel Model}

We consider a Gaussian broadcast channel with $n$ homogeneous users, a transmitter with $M$ antennas and receivers equipped with $N$ antennas. We also assume a block fading model for the channel. Therefore we may write the received vector at the $i$ 'th receiver as, $Y_{i}=H_{i} S+N_{i}$ for $i=1, \ldots, n$ where $H_{i}(N \times M)$ represents the channel, $N_{i}$ is the $N \times 1$ noise vector. Both $H_{i}$ 's and $N_{i}$ 's have i.i.d. complex Gaussian distribution with zero mean and the variance of one. Furthermore, the average power constraint of the input signal implies that $\operatorname{tr}\left\{E\left(S S^{*}\right)\right\} \leq P$, where $P$ is the total average transmit power which is assumed to be fixed.

\section{Scaling LAWs for Different Schemes}

It is clear that, by only sending to the user with maximum capacity, the sum rate throughput of time-sharing (denoted by $R_{t s}$ ) can be written as [5],

$$
R_{t s}=\max _{i=1, \ldots, n} C\left(H_{i}, P\right)=\max _{i=1, \ldots, n} \log \operatorname{det}\left(I+P H_{i} H_{i}^{*}\right) .
$$

${ }^{1}$ This work was supported in part by NSF Grant CCR-0133818, by the office of Naval Research Grant N00014-02-1-0578, and By Caltech's Lee Center for Advanced Networking.
Lemma 1. (time-sharing) For $M, N$, and $P$ fixed, we have,

$$
\lim _{n \rightarrow \infty} \frac{E\left\{R_{t s}\right\}}{\min (M, N) \log \log n}=1,
$$

and if $M$ logarithmically increases with $n$, we have

$$
\lim _{n \rightarrow \infty} \frac{E\left\{R_{t s}\right\}}{N \log \log n}=1 .
$$

Lemma 2. (dirty paper coding) Let $R_{D P C}$ denote the sum rate capacity of MIMO Gaussian broadcast channel achieved by DPC. For $M$ and $P$ fixed and any $N$, we have,

$$
\lim _{n \rightarrow \infty} \frac{E\left\{R_{D P C}\right\}}{M \log \log n N}=1 .
$$

For $M=\beta \log n$ and $N$ and $P$ fixed, we have,

$$
\lim _{n \rightarrow \infty} \frac{E\left\{R_{D P C}\right\}}{M}=\gamma
$$

where $\gamma, \beta$ and $\alpha$ are constants independent of $n$. Furthermore, we can bound $\gamma$ by $\gamma \leq \log (1+\alpha)$ where $\alpha$ is the unique solution to $\alpha-\beta \log \alpha=1+\beta-\beta \log \beta$.

Therefore, the asymptotic gain of DPC over time-sharing for the sum rate is $\frac{M}{\min (M, N)}$ when $M$ and $N$ are fixed.

Lemma 3. (beamforming) Let $M, N$, and the total average transmit power be fixed, and $R_{B F}$ denotes the sum rate of beamforming. Then

$$
\lim _{n \rightarrow \infty} \frac{E\left\{R_{B F}\right\}}{M \log \log n N}=1 .
$$

Furthermore, if $M=\frac{\log n+3 \log \log n}{\frac{c}{P}+\log (1+c)}$ where $c$ is a constant, then

$$
\lim _{n \rightarrow \infty} \frac{E\left\{R_{B F}\right\}}{M}=\gamma^{\prime},
$$

where $\gamma^{\prime}$ is a constant less than $\gamma$ in Lemma 2 and larger than $\log (1+c)$.

\section{REFERENCES}

[1] G. Caire and S. Shamai, "On the achievable throughput of a multi-antenna Gaussian broadcast channel," IEEE Trans. Inform., vol. 49, no. 7, pp. 1691-1706, July 2003.

[2] P. Viswanath and D. N. Tse, "Sum capacity of the vector Gaussian broadcast channel and downlink-uplink duality," IEEE Trans. Inform., vol. 49, no. 8, pp. 1912-1921, Aug. 2003.

[3] S. Vishwanath, N. Jindal, and A. Goldsmith, "Duality, achievable rates and sum rate capacity of Gaussian MIMO broadcast channel," IEEE Trans. Inform., vol. 49, no. 10, pp. 2658-2668, Oct. 2003

[4] W. Yu and J. Cioffi, "Sum capacity of Gaussian vector broadcast channel," submitted to IEEE Trans. Info. Theory, 2001.

[5] N. Jindal and A. Goldsmith, "Dirty paper coding vs. TDMA for MIMO broadcast channel," Proc. IEEE ICC, June 2004.

[6] M. Sharif and B. Hassibi, "Scaling laws of sum rate of time sharing, DPC, and beamforming for MIMO BC with Many Users," submitted to IEEE Trans. comm., 2004. 